\title{
"Negative magnetic buoyancy" effects and reconstruction of toroidal field in the solar convection zone
}

\author{
Valery N. Krivodubskij \\ Astronomical Observatory of Kyiv National Taras Shevchenko University \\ Observatorna Street 3, Kyiv, 04053, Ukraine, email: krivod1@observ.univ.kiev.ua
}

\begin{abstract}
This investigation is devoted to study the turbulent convective transport of mean (large-scale) magnetic field in the solar convection zone (SCZ). For the SCZ model by Stix (1989) the reconstruction of the toroidal field was calculated as a result of the balance of mean-field magnetic buoyancy and two "negative magnetic buoyancy" effects: i) macroscopic turbulent diamagnetism and ii) the $\nabla \rho$ effect. It is shown that at high latitudes negative buoyancy effects block the magnetic fields, about $3000-4000 \mathrm{G}$, near the bottom of the SCZ. This may be the most plausible reason why a deep-seated field here could not become as apparent at the solar surface as sunspots. However, at the near equatorial domain in the deep layers the $\nabla \rho$ effect, with allowance for rotation, causes the upward magnetic transport, that facilitates penetration of strong fields to the surface where they emerge as sunspot patters in the "royal zone".
\end{abstract}

For the excitation of toroidal magnetic field by the Sun's differential rotation stretching a weak poloidal field to be effective, it is necessary that magnetic flux tubes be kept in the generation region in the SCZ for a long time. However, due to efficient magnetic buoyancy it is generally difficult to ensure significant magnetic amplification during a time comparable to the solar cycle period. Thus, some "negative magnetic buoyancy" effects are required to compensate for the loses of magnetic flux in generation zone. The most encouraging magnetic transport phenomena which may play antibuoyancy role include the macrocsopic diamagnetism (Zeldovich 1956), and the pumping of the magnetic flux caused by the inhomogeneity of fluid density in the turbulent plasma (Drobyshevskij 1977; Vainshtein 1983). Here we examined whether these advection effects really suppress the magnetic buoyancy in the SCZ and attempt to clarify in what way they may promote penetration of the magnetic field to the surface at the sunspot belt.

The physical sense of macroscopic diamagnetism consists in an expulsion of the mean magnetic field along the gradient of the turbulent viscosity $\nu_{\mathrm{T}} \approx\left(\frac{1}{3}\right) v l \approx\left(\frac{1}{3}\right) \tau v^{2}(v, l$ and $\tau$ are the r.m.s. velocity, mixing length and correlation time of the turbulence) with the effective velocity $\vec{V}_{\mu}=-\nabla \nu_{\mathrm{T}} / 2$ (Zeldovich, Ruzmaikin \& Sokoloff 1983). Our calculations for the SCZ model by Stix (1989) had shown that the radial profile of $\nu_{\mathrm{T}}(z)$ is a convex function with maximum value, $\nu_{\text {Tmax }} \approx 2 \cdot 10^{13} \mathrm{~cm}^{2} / \mathrm{s}$, located at the depth $z \approx 130 \mathrm{Mm}$ (Fig. 1a). Therefore, the radial inhomogeneity of $\nu_{\mathrm{T}}(z)$ causes an essential vertical magnetic advection. In the lower part of the SCZ the turbulent diamagnetism acts against magnetic buoyancy, displacing the horizontal fields downwards (Fig. 1b). The global magnetic field suppresses the macroscopic diamagnetism, so in the nonlinear regime transfer velocity depends on magnetic field, $V_{\mathrm{D}}(\beta)=6 V_{\mu} \Psi_{\mathrm{D}}(\beta)$ (Kitchatinov \& Rüdiger 1992). Here $\beta=B / B_{\text {eq }}$ is the magnetic field normalized to the energy equipartition value $B_{\mathrm{eq}} \approx(4 \pi \rho)^{1 / 2} v, \Psi_{\mathrm{D}}(\beta) \approx(1 / 6)-\beta^{2} / 5$ is the quenching function for the weak fields case, $\rho$ is the plasma density. Assuming balance between the buoyant and downward diamagnetic transports, one can determine the value of the steady-state field $B_{\mathrm{S}}$, 

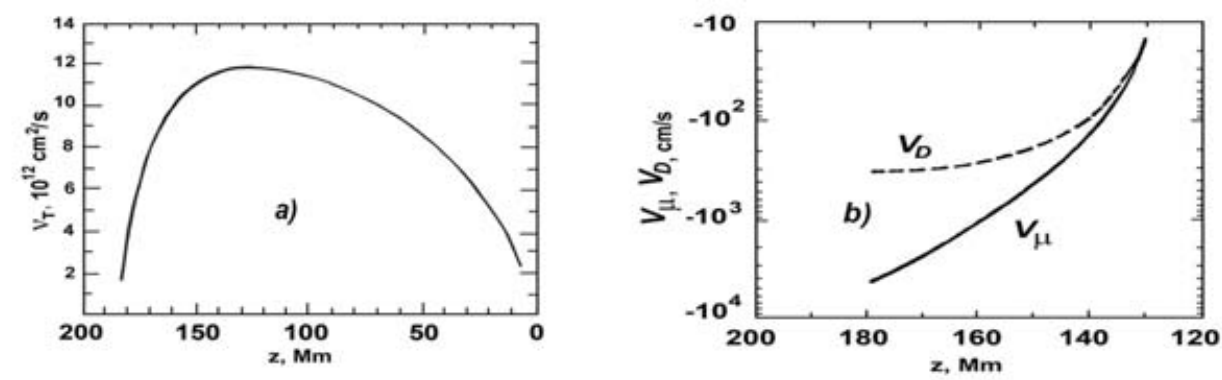

Figure 1. a) Radial profile of the turbulent viscosity coefficient, $\nu_{\mathrm{T}} \approx\left(\frac{1}{3}\right) v l$, in the SCZ; b) Distributions of the downward velocities of macroscopic diamagnetic transport with depth, $z$, in the lower part of the SCZ: the velocity $V_{\mu}$ results from the kinematic approach (solid curve), while $V_{\mathrm{D}}\left(\beta_{\mathrm{S}}\right)$ is supplied by a nonlinear theory (dotted curve).
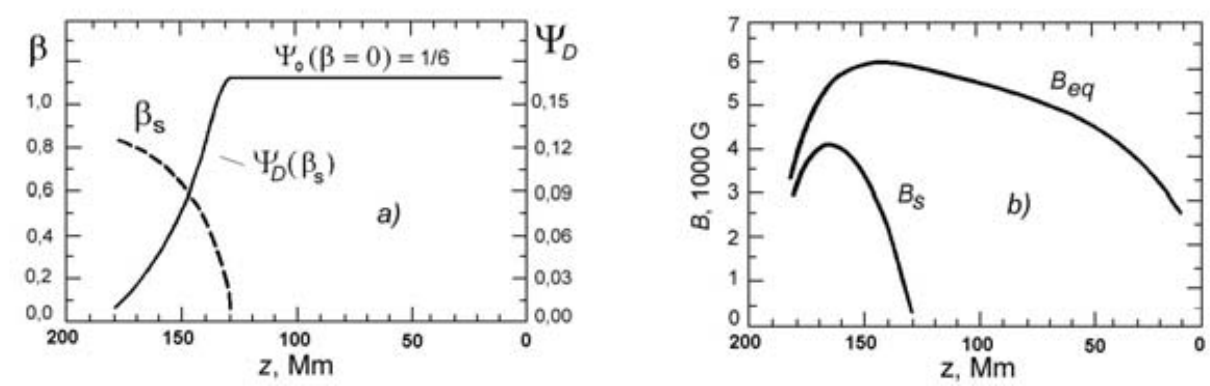

Figure 2. a) Radial distribution of the parameter of the normalized steady-state magnetic field, $\beta_{\mathrm{S}}=B_{\mathrm{S}} / B_{\text {eq }}$, and the magnetic quenching function, $\Psi_{\mathrm{D}}\left(\beta_{\mathrm{S}}\right) \approx(1 / 6)-\beta_{\mathrm{S}}^{2} / 5$, in the SCZ; b) Distributions of the steady-state magnetic field, $B_{\mathrm{S}}=\beta_{\mathrm{S}} B_{\text {eq }}$, and the equipartition magnetic field, $B_{\text {eq }} \approx(4 \pi \rho)^{1 / 2} v$, with depth, $z$. It can be seen that the macroscopic diamagnetism promotes the formation of the magnetic layer, $B_{\mathrm{S}} \approx 3000-4000 \mathrm{G}$, near the bottom of the SCZ, $\mathrm{z} \approx$ 150-180 Mm.

for which the bouyant rise is compensated for by the diamagnetic submersion. Taking into account the mean-field magnetic buoyant velocity $V_{\mathrm{B}}(\beta) \approx\left(\frac{l}{H_{P}}\right)\left(\frac{v}{\gamma}\right) \frac{\beta^{2}}{15}\left(H_{P}\right.$ is the pressure scale, $\gamma=5 / 3$ is the adiabaticity index) (Kitchatinov \& Pipin (1993)), we found that nonlinear downward transfer velocity $V_{\mathrm{D}}\left(\beta_{\mathrm{S}}\right)$ in the lower part of the SCZ (Fig. 1b) is sufficient to resist the magnetic buoyant velocity. As a result, the turbulent diamagnetism promotes near the SCZ the storage of the steady-state toroidal magnetic field $B_{\mathrm{S}}=\beta_{\mathrm{S}} B_{\text {eq }}$ about $3000-4000 \mathrm{G}$ (Fig.2a, Fig.2b).

Another macroscopic transport effect, which is caused by inhomogenity of material density, may arise in turbulent plasma under certain condition (Drobyshevskij 1977; Vainshtein 1983). This magnetic transport can be intrpreted as follows. The amplitude of the magnetic fluctuations $\vec{b}$, produced by turbulent pulsations $\vec{v}$, is increased in a direction of the growth of the fluid density: $b^{2} \approx 4 \pi \rho v^{2}$. At the same time, the random electric currents, $\vec{j}=(c / 4 \pi)$ curl $\vec{b}$, also are increasing in this direction. These modified currents change the initial distribution of the large-scale field. As a result, the net distribution of the mean magnetic field, $\vec{B}$, in the non-linear regime is equivalent to field transfer with the velocity $\vec{V}_{\rho} \approx\left(\frac{1}{6}\right) \tau v^{2}\left(\frac{\nabla \rho}{\rho}\right)$ (Kitchatinov 1982 ). We call it the " $\nabla \rho$ " effect. Since the plasma density varies about by six orders of magnitude over a vertical extent of the SCZ, a very strong downward magnetic pumping appears here (Fig. 3a). The rotation 

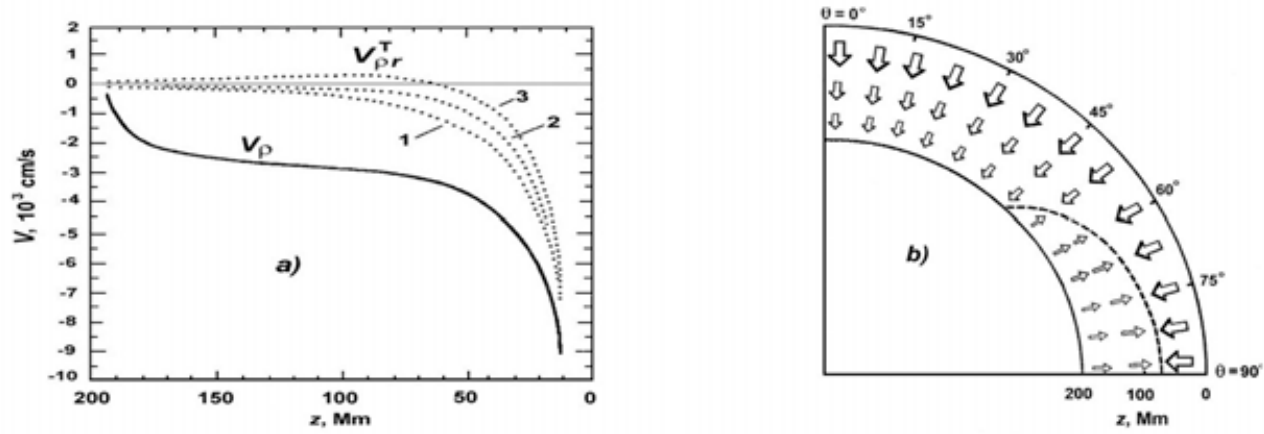

Figure 3. a) Radial prifiles of the transport velocity $V_{\rho}$ (solid line) and the transport velocity of toroidal magnetic component $V_{\rho \mathrm{r}}^{\mathrm{T}}$ (dotted lines) in the SCZ for different colatitudes $\theta$ : $1-\theta=0^{\circ}$ (pole), $2-\theta=45^{\circ}, 3-\theta=90^{\circ}$ (equator)). Negative velocities correspond to downwards transport; b) A meridional cross section of the SCZ which shows the distribution of the radial transport velocity of toroidal field $V_{\rho \mathrm{r}}^{\mathrm{T}}(z, \theta)$ along depth $z$ and colatitude $\theta$. The arrows denote the direction of transport.

imparts new properties to the $\nabla \rho$ magnetic transport. Under the Sun's rotation the $\nabla \rho$ effect realizes the "fields selection", that results in an independent transfer of the azimuthal and meridional field components (Kitchatinov 1991). The radial transport of the azimuthal (toroidal) field, $\vec{B}_{\mathrm{T}}$, is of main interests because this field is involved in the origin of sunspot on the surface. The corresponding transport velocity depends on depth $z$ and colatitude $\theta: \vec{V}_{\rho r}^{\mathrm{T}}(z, \theta) \approx 6 \vec{V}_{\rho}(z)\left\{\varphi_{2}[\omega(z)]-\sin ^{2} \theta \varphi_{1}[\omega(z)]\right\}$ (Kitchatinov 1991). The functions $\varphi_{1}(\omega)$ and $\varphi_{2}(\omega)$, depending on the Coriolis number $\omega=2 \tau \Omega$, describe the rotation effect on turbulent convection, $\Omega$ is the angular velocity. Depending on the sign of $\left(\varphi_{2}[\omega(z)]-\sin ^{2} \theta \varphi_{1}[\omega(z)]\right)$, magnetic transport may be directed downward (when this sign is positive) and upward (when the sign is negative). This property produces a complicated distribution of the transport velocity $V_{\rho \mathrm{r}}^{\mathrm{T}}(z, \theta)$ over the SCZ (Fig. 3b).

The reconstruction of the toroidal field in the SCZ was examined assuming the balance between mean-field magnetic buoyancy, turbulent diamagnetism and the rotationally modified $\nabla \rho$ effect (Krivodubskij 2005). We found that two negative buoyancy phenomena may be the most plausible reason why the polar deep-rooted toroidal field could not become apparent at the surface as sunspots at high latitudes. At the same time, at mid to low latitudes in the deep layers the upward $\nabla \rho$ advection can facilitate strong magnetic fields to emerge through the surface at the sunspot belt.

\section{References}

Drobyshevskij, E.M. 1977 Astrophys. Space Sci. 46, 41-49.

Kitchatinov, L.L. 1982 Magnitnaja Hidrodinamika (Riga) 3, 67-73.

Kitchatinov, L.L. 1991 Astron. Astrophys. 243, 483-491.

Kitchatinov, L.L. \& Pipin, V.V. 1993 Astron. Astrophys. 274, 647-652.

Kitchatinov, L.L. \& Rüdiger, G. 1992 Astron. Astrophys. 260, 494-498.

Krivodubskij, V.N. 2005 Astron. Nachr. 326, 64-71.

Stix, M. 1989 The Sun, p. 200. Verlag, Berlin.

Vainshtein, S.I. 1983 Magnitnye Polia v Kosmose, Nauka, Moscow.

Zeldovich, Y.B. 1956 Zh. Eksp. Teor. Fiz. (Moscow) 31, 154.

Zeldovich, Y.B., Ruzmaikin, A.A., \& Sokoloff, D.D 1983 Magnetic Fields in Astrophysics, Gordon and Breach, New York. 